\title{
Current limiting characteristics of superconducting fault current limiter with parallel and series connection in experimental power system
}

\section{$\operatorname{AUTHOR}(S)$ :}

Shirai, Y; Mochida, A; Shiotsu, M; Hatta, H; Oide, T; Nitta, $T$

\section{CITATION:}

Shirai, Y...[et al]. Current limiting characteristics of superconducting fault current limiter with parallel and series connection in experimental power system. IEEE TRANSACTIONS ON APPLIED SUPERCONDUCTIVITY 2004, 14(2): 871-874

\section{ISSUE DATE:}

2004-06

URL:

http://hdl.handle.net/2433/50262

\section{RIGHT:}

(c)2004 IEEE. Personal use of this material is permitted. However, permission to reprint/republish this material for advertising or promotional purposes or for creating new collective works for resale or redistribution to servers or lists, or to reuse any copyrighted component of this work in other works must be obtained from the IEEE. 


\title{
Current Limiting Characteristics of Superconducting Fault Current Limiter With Parallel and Series Connection in Experimental Power System
}

\author{
Yasuyuki Shirai, Member, IEEE, Akihiro Mochida, Masahiro Shiotsu, Hiroyuki Hatta, Tomomi Oide, and \\ Tanzo Nitta, Member, IEEE
}

\begin{abstract}
A trial three-phase SCFCL with adjustable trigger current level was proposed and fabricated. Current limiting characteristics of the SCFCL with parallel and series connection using two of the three-Phase SCFCLs was investigated experimentally in a model power system consisting a small synchronous generator and an artificial parallel transmission lines. The pair of the SCFCL was installed in one of three phases of the fault line. The fault current limiting characteristics of the pair of SCFCL at one line grounded ( 1 LG) fault was studied with various fault phases. In every test case, the SCFCL limited the fault current below a certain level. In the series-connection cases, which one or both of the pair of SCFCLs turn into the current limiting mode can be controlled by calibrating the trigger current level of each SCFCL. It was observed that it depends on the fault phase. In the parallel connection cases, both of the pair of SCFCLs turn into current limiting mode nearly simultaneously in every fault phase.
\end{abstract}

Index Terms-Power system characteristics, series/parallel connection, superconducting fault current limiter.

\section{INTRODUCTION}

$\mathbf{S}$ UPERCONDUCTING fault current limiters (SCFCLs) of various types have been proposed and tested [1]-[3]. However, there are few investigations from the viewpoint that the SCFCL is one of the power system apparatus. It is important to study the power system characteristics of the SCFCLs. The most significant features of the SCFCLs requested from the power system operating conditions are a limiting impedance, a trigger current level and a recovery time. In order to meet these demanded features, it is expected to design SCFCL unit with series-connection for larger limiting impedances or parallel-connection for larger rated current and reliability. However, it is not so easy to fabricate number of SCFCLs of exactly same specifications for series/parallel connection.

Manuscript received October 20, 2003. This work was supported in part by the 21st Century COE Program 14219201, the MEXT Japan under 21st Century COE Project 14219201.

Y. Shirai, A. Mochida, and M. Shiotsu are with the Department of Energy Science and Technology, Kyoto University, Kyoto 606-8501, Japan (e-mail: shirai@energy.kyoto-u.ac.jp; mochida@pe.energy.kyoto-u.ac.jp; shiotsu@ energy.kyoto-u.ac.jp).

H. Hatta was with the Department of Electrical Engineering, the University of Tokyo, Tokyo, 113-8656. He is now with CRIEPI, Japan (e-mail: hitta@criepi.denken.or.jp).

T. Oide and T. Nitta are with the Department of Electrical Engineering, University of Tokyo, Tokyo, 113-8656, Japan (e-mail: oide@asc.t.u-tokyo.ac.jp; nitta@asc.t.u-tokyo.ac.jp).

Digital Object Identifier 10.1109/TASC.2004.830305

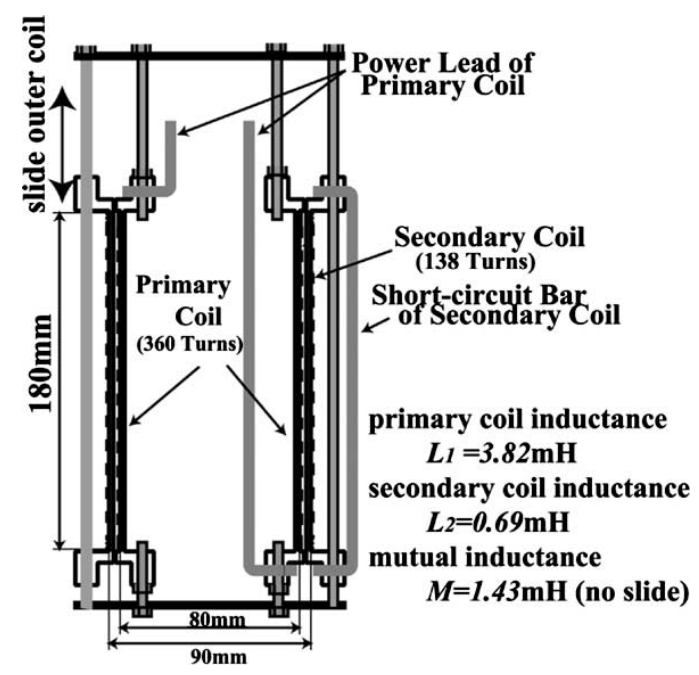

Fig. 1. One of the test 3-phase SCFCL with adjustable trigger current.

In this paper, current limiting characteristics of the SCFCL with parallel or series connection using two SCFCLs is investigated experimentally in a model power system consisting of a small synchronous generator and an artificial parallel transmission lines (that is, a simulated fault line and a nonfault line). A 3-phase SCFCL of transformer type, which can calibrate the trigger current level, independently for each phase, was designed, made and have been tested in an artificial transmission line system [4], [5]. Excellent recovery characteristics was confirmed and discussed [6], [7]. Two of the three-phase SCFCLs were used in the experimental studies. The pair of SCFCL was installed in one of three phases of the fault line. The fault current limiting characteristics of the pair of SCFCL at one line grounded (1 LG) fault was studied with various fault phases.

\section{EXPERIMENTAL SYSTEM}

\section{A. Test 3-Phase SCFCL}

The three-phase SCFCL unit contains three SCFCLs of transformer type in one cryostat [4]. As shown in Fig. 1, one of the SCFCL consists of two superconducting coils magnetically coupled co-axially. The inner (primary) coil will be connected to a power line. The outer (secondary) coil is superconductively short-circuited. The primary coil can be slided with small slide 


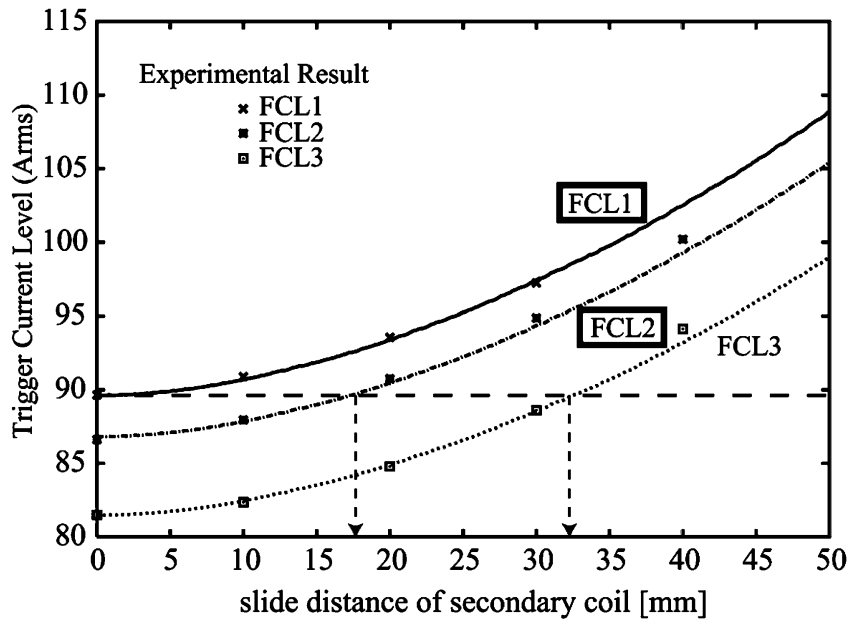

Fig. 2. Trigger current level of test 3-phase SCFCLs and calibration characteristics by sliding the outer (secondary) coil.

distance in order to calibrate the trigger current level. When both superconducting coils are in the superconducting state, the magnetic flux is cancelled and the reactance of the SCFCL seen from the system is small (leakage reactance). When the fault current reaches the trigger current level, the super-normal transition occurs only in the secondary coil. The reactance of the SCFCL becomes large (almost equal to that of the primary coil) and the fault current is limited.

The superconducting wire of the primary coil is made of twisted three $\mathrm{CuNi} / \mathrm{Cu} / \mathrm{NbTi}$ (3.3:0.3:1) strands. The secondary coil wire is made of $\mathrm{CuNi} / \mathrm{NbTi}$ (4.3:1) strands.

\section{B. Trigger Current Level and Its Calibration}

The designed trigger current level without calibration is 85.7 $\mathrm{A}_{\mathrm{rms}}$. The trigger current level of each SCFCL can be calibrated by sliding the outer (secondary) coil. Fig. 2 shows the trigger current level of each SCFCL measured by increasing the current slowly, that is, quasisteadily. Measured trigger current of each SCFCL without slide was $89.7 \mathrm{~A}_{\mathrm{rms}}$ for SCFCL of no. 1 (FCL1), $86.62 \mathrm{~A}_{\mathrm{rms}}$ for that of no. 2 (FCL2) and $81.65 \mathrm{~A}_{\mathrm{rms}}$ for that of no. 3 (FCL3), respectively. Although three SCFCL was designed and made with same parameters and materials, the trigger current level of each SCFCL was different each other around $10 \%$.

It is expected that it is not so easy to make a number of SCFCLs of exactly same characteristics. Therefore, we proposed the SCFCL whose trigger current level can be calibrated after fabrication.

As seen from Fig. 2, the trigger current level of three SCFCLs can be set the same value of $89.7 \mathrm{~A}_{\text {rms }}$ (the instantaneous value of $126.8 \mathrm{~A}$ ) by sliding outer coil of FCL2 by $18 \mathrm{~mm}$, and that of FCL 3 by $32 \mathrm{~mm}$, respectively. It should be more important in the series-connecting operation of SCFCLs. FCL1 and FCL2 were used in all the experiments of series- or parallel-connecting operations.

\section{Model Power System Including SCFCLs}

Fig. 3 shows an experimental system. A 3-phase synchronous generator was connected to an infinite bus $(210 \mathrm{~V}$ commercial

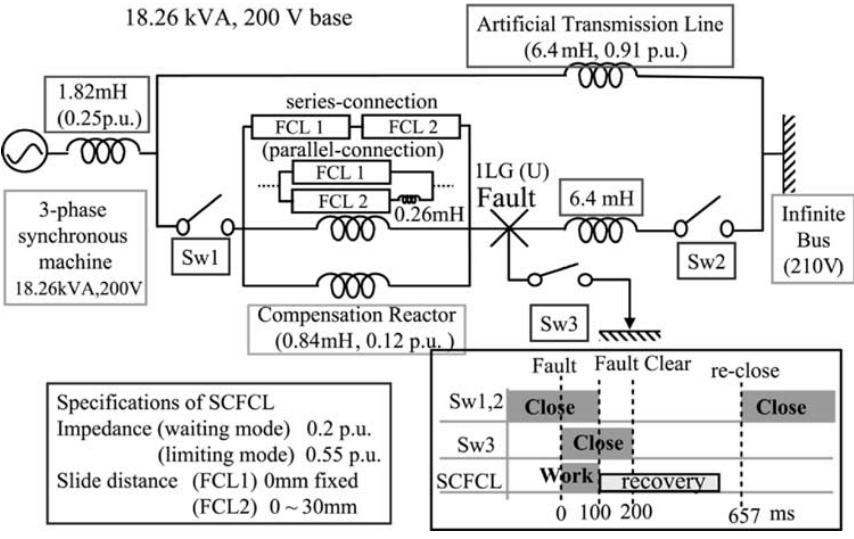

Fig. 3. One machine infinite bus transmission system with series-connected SCFCL and the switching sequence for the simulated fault conditions.

power line) through parallel artificial transmission lines. The lower line (fault line) has Sw3 in parallel for simulated fault and has circuit breakers (Sw1 and Sw2) in series. The series- or parallel-connected SCFCL (no. 1 and no. 2) was installed between the fault point and the Sw3 only in u-phase line and small compensation reactors $(0.84 \mathrm{mH})$ were inserted in the other phase lines. In the parallel-connection cases, a small reactor of 0.26 $\mathrm{mH}$ was set in series with FCL2 to compensate the leakage reactance. The ratings of the generator is $18.26 \mathrm{kVA}$ of capacity, $200 \mathrm{~V}$ of voltage, 52.5 A of current, 19.2 A of field current, $1500 \mathrm{rpm}$ of rotating speed, 4-poles, $50 \mathrm{~Hz}$. The field current is supplied by a constant voltage source. Base ratings of per unit (p.u.) value are $18.26 \mathrm{kVA}, 200 \mathrm{~V}$. The generator was connected to the transmission line through a reactor of $1.82 \mathrm{mH}$ for the series-connection case and of $1.3 \mathrm{mH}$ for the parallel-connection case.

All switches are magnetically controlled. The switching sequence is shown in Fig. 3. Sw1 and Sw2 are normally closed so that the generator output power flows through the double transmission lines. At a certain time, the Sw3 is closed to simulate the 1-line (u-phase) grounded fault, then the large fault current flows through the SCFCLs. The SCFCLs start to reduce the fault current immediately. At $100 \mathrm{~ms}$ after the fault, Sw1 and Sw2 are open to reject the fault line. After the fault was removed, Sw1 and Sw2 are re-closed to be back to the initial condition. During the fault line rejection time $(577 \mathrm{~ms})$, the SCFCLs should recover to the waiting mode. The fault phase, which means that the phase of the FCL current at the fault occurrence, was changed from 0 to 180 degrees by use of sequence controller.

The each SCFCL was equipped with $\mathrm{ZnO}$ device (varistor voltage is $400 \mathrm{~V}$ ) in parallel to suppress the over-voltage [8].

The generator output was set about $6 \mathrm{~kW}$ and the terminal voltage was $200 \mathrm{~V}$. The voltage $v_{F C L}$ across and the currents $i_{F C L}$ through each SCFCLs of series-connected or parallel-connected were measured at the fault for various fault phases.

\section{EXPERIMENTAL RESULTS AND DISCUSSION}

\section{A. Current Limiting by Series-Connected SCFCL}

The fault current limiting tests were carried out with two series-connected SCFCLs at 1 LG fault of different fault phases. The slide distance of FCL2 was changed between $0 \mathrm{~mm}$ and 

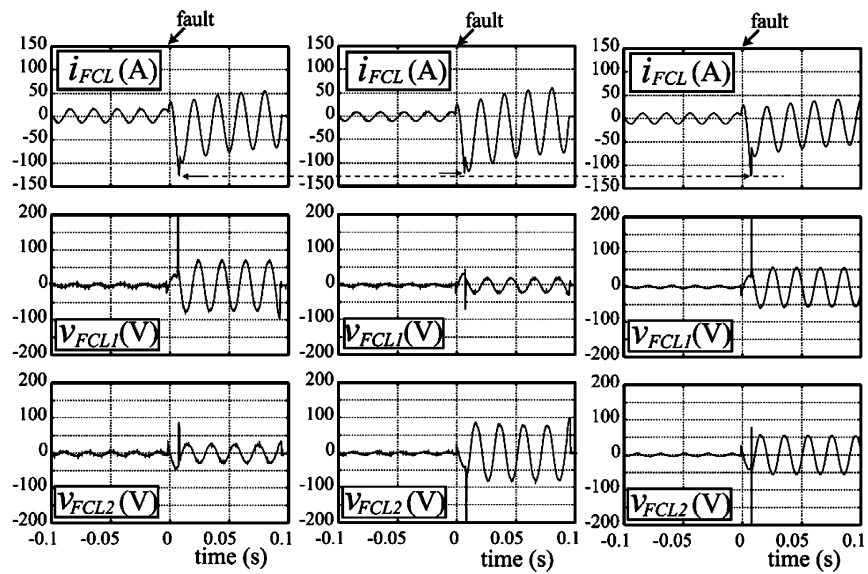

(a) FCL1 works slide distance $=30.0 \mathrm{~mm}$ (b) FCL2 works slide distance $=18.0 \mathrm{~mm}$ slide distance $=24.4 \mathrm{~mm}$ (c) Both work fault phase $=142$ degree

Fig. 4. Current and voltages of each FCLs (series-connected) at the fault. (a) Only FCL1 works with $30 \mathrm{~mm}$, (b) only FCL2 works with $18 \mathrm{~mm}$, (c) both of them work with $24.4 \mathrm{~mm}$ of the slide distance, respectively. Fault phase is 142 degree.

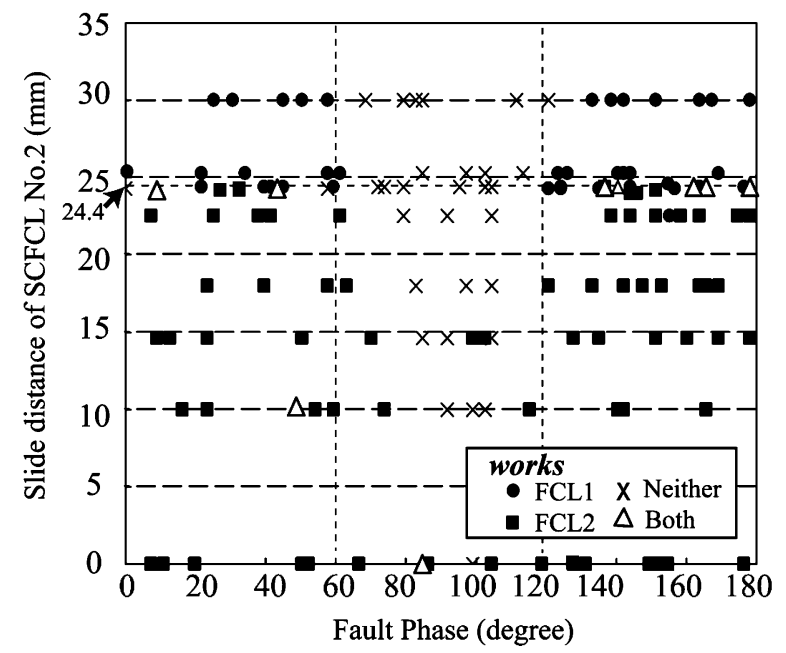

Fig. 5. Experimental results classified as four cases with the slide distance of FCL2 and the fault phases.

$30 \mathrm{~mm}$. Throughout the experiments, the FCLs recovered from the current limiting mode to the waiting mode successfully with isolation time of $577 \mathrm{~ms}$. The experimental results were classified as four cases, that is, 1) only FCL1 works, 2) only FCL2 works, 3) both FCLs work simultaneously and 4) neither works. Fig. 4 shows one of the experimental results of current and voltages of each SCFCL with slide distance of FCL2 of $30 \mathrm{~mm}$ (FCL1 works), with that of $18 \mathrm{~mm}$ (FCL2 works) and with that of $24.4 \mathrm{~mm}$ (both work). The fault phase is 142 degree. As soon as the fault current reached about $125 \mathrm{~A}$, both of FCLs or only FCL1 started to reduce the current successfully as shown in Figs. 4(a) and (c). The trigger current was slightly smaller in case with the slide distance was $18 \mathrm{~mm}$ in Fig. 4(b). The limited fault current when the both FCLs worked was reduced more than that in case that one of the FCLs worked.

The four cases of the experimental results were categorized on the fault phase and the slide distance plane as shown in Fig. 5.

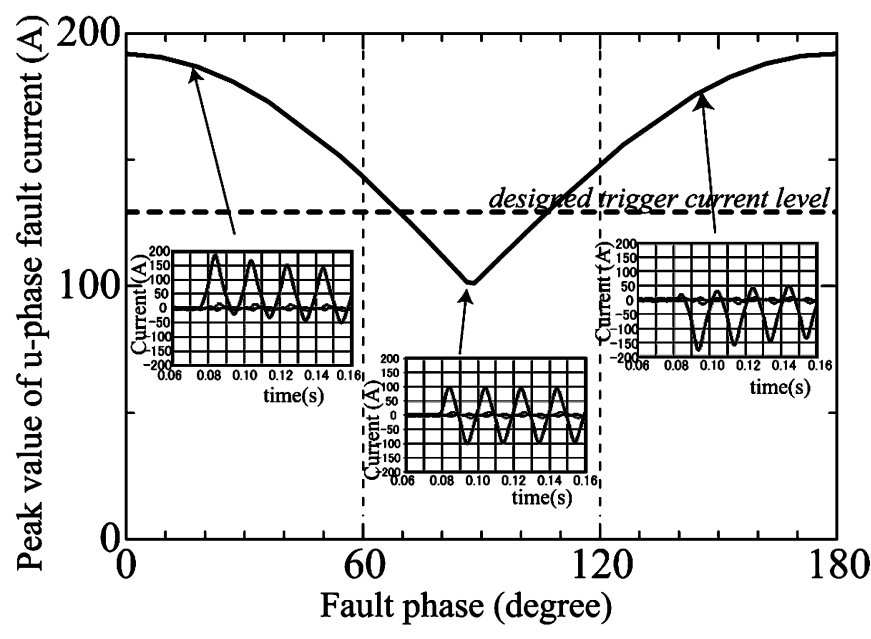

Fig. 6. Peak value of the fault current for fault phase without SCFCLs (simulation result).

As seen from Fig. 2, the trigger current level of FCL2 is smaller than that of FCL1 with the slide distance of less than $18 \mathrm{~mm}$. The experimental results (Fig. 5) show that only FCL2 turned into the current limiting mode in almost all the test cases with the slide distance of less than $22.5 \mathrm{~mm}$. On the other hand, only FCL1 worked with the slide distance of more than $25.3 \mathrm{~mm}$. All the four cases appeared with the slide distance of $24.4 \mathrm{~mm}$, that is, it was considered that the each trigger current level of seriesconnected FCLs was calibrated to be almost the same. The slide distance of $24.4 \mathrm{~mm}$ on the boundary was slightly different from that of $18 \mathrm{~mm}$ obtained in the quasisteady state condition as shown in Fig. 2. The possible cause is that the trigger current level of SCFCL is affected by the differential of fault current, which depends on the fault phases.

Fig. 6 shows the peak value of the fault current without a SCFCL as a function of fault phase obtained by computer simulation. Typical waveforms of the fault current for each fault phase were also shown. DC component of the fault current varies with fault phase. The broken line indicates the designed trigger current level of FCL1. The peak value of the fault current is less than the designed trigger current level with the fault phase of around 90 degrees. Therefore, neither of FCLs worked for fault phase of around 90 degrees as shown in Fig. 5.

The actual trigger currents measured from the experimental results are shown in Fig. 7 for the fault phase with various slide distances as a parameter. Each line indicates a polynomial interpolation of the second order of the trigger currents with each slide distance obtained by the least-square method. The trigger current becomes larger with increasing the slide distance up to $24.4 \mathrm{~mm}$. The upper three lines for the slide distance of more than $24.4 \mathrm{~mm}$ are almost equal each other independent of the slide distance of FCL2, because they are determined by trigger current level of FCL1.

The trigger current level decreases as the fault phase approaches the zero-cross point ( 0 and 180 degree) for all cases of slide distances. This is because the trigger current level decreases for larger differential of the fault current.

It was confirmed that the trigger current level of series-connected FCLs can be calibrated by the proposed SCFCL. 


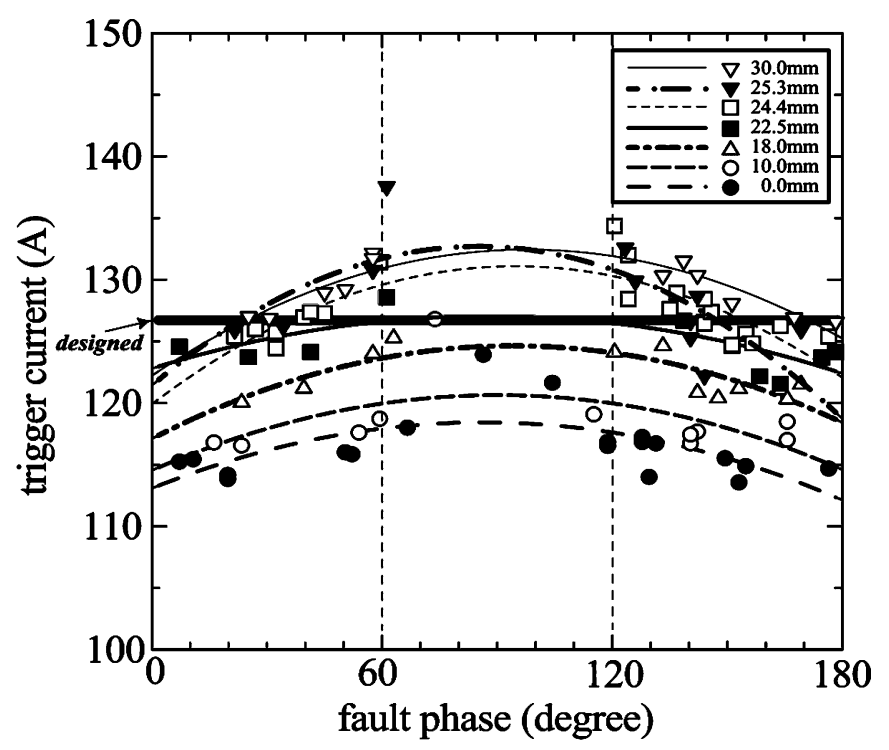

Fig. 7. Experimental results of trigger current for fault phase with slide distances between $0 \mathrm{~mm}$ and $30 \mathrm{~mm}$ as a parameter.
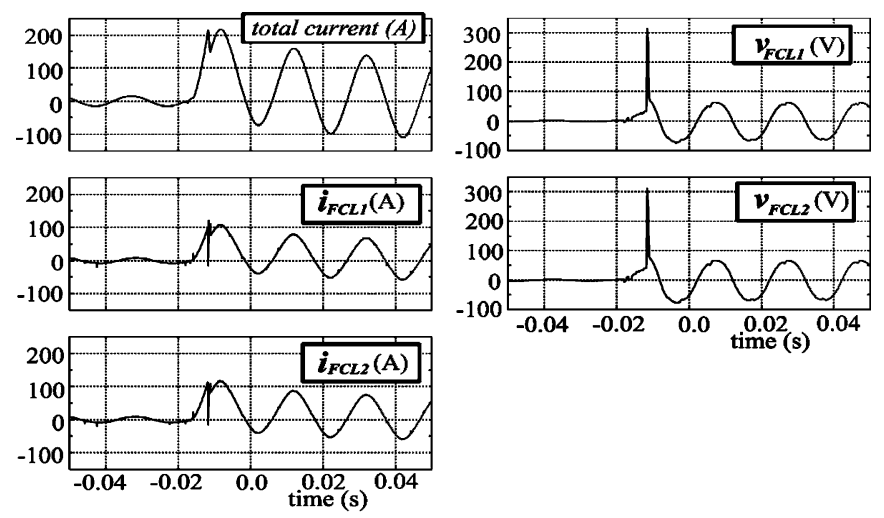

Fig. 8. Experimental result of fault current limiting operation by parallel-connected FCLs at 1 LG fault.

\section{B. Current Limiting by Parallel-Connected SCFCL}

The series-connected SCFCL was changed to be parallel-connected one and installed at the same place. Fig. 8 shows one of the experimental results of the voltages and currents of each FCLs and the total current of $\mathrm{u}$-phase at $1 \mathrm{LG}$ fault. The slide distance of FCL2 was $16.6 \mathrm{~mm}$ and the fault phase was $28.8 \mathrm{de}$ gree.

At first, FCL2 started to limit the fault current and the fault current rushed to FCL1. FCL1 turned into the current limiting mode immediately. As seen from the total current waveform, the current limiting operation started at once and the trigger current level was almost twice larger than that of each FCL. The trigger current level was determined by lower one of each FCL (FCL2 for this test case). In all test cases, both of the parallel FCL started to limit the fault current simultaneously.

\section{CONCLUSION}

Fault current limiting characteristics of the SCFCL with series or parallel connection using two SCFCLs were investigated experimentally in a model power system. A pair of SCFCL with adjustable trigger current level was installed in one of three phases of the fault line and was tested at one line grounded (1 LG) fault with various fault phases. The following results were obtained:

1) It was observed that, in the series connection case, which one or both of the pair of SCFCLs turn into the current limiting mode depends on the fault phase;

2) Trigger current (fault current at the initiation of current limiting) can be calibrated to a certain extent by use of the proposed SCFCL in series or parallel connecting operations;

3) It was found that the trigger current depends on the fault phase. It is expected that not only the fault current value but also the differential of it affect the trigger current. That is, the larger differential of the current makes the trigger current lower;

4) In the parallel connection case, both of the pair of SCFCLs turn into current limiting mode nearly simultaneously in every fault phases.

\section{REFERENCES}

[1] H. J. Boenig and D. A. Paice, "Fault current limiter using a superconducting coil," IEEE Trans. Magnetics, vol. 19, no. 3, pp. 1051-1053, May 1983

[2] B. Gromoll et al., "Resistive fault current limiters with YBCO films-100 kVA functional model," IEEE Trans. Appl. Supercond., vol. 9, no. 2, pp. 656-659, June 1999.

[3] E. Leung et al., "Design \& development of a $15 \mathrm{kV}, 20 \mathrm{kA}$ HTS fault current limiter," IEEE Trans. Appl. Supercond., vol. 10, no. 1, pp. 832-835, March 2000.

[4] H. Hatta, T. Nitta, S. Muroya, Y. Shirai, and T. Kitagawa, "Experimental study on sudden-short-circuit characteristic of synchronous generator with SCFCL," IEEE Trans. Appl. Supercond., vol. 11, no. 1, pp. 2343-2346, March 2001.

[5] Y. Shirai et al., "A proposal of new operating procedure of transformer type fault current limiter," IEEE Trans. Appl. Supercond., vol. 12, no. 1, pp. 885-889, March 2002.

[6] - "Study on recovery time of a superconducting fault current limiter with adjustable trigger current level," IEEE Trans. Appl. Supercond., vol. 11, no. 1, pp. 2086-2089, March 2001.

[7] —, "Recovery characteristics of fault current limiter with adjustable trigger current level," IEEE Trans. Appl. Superconduct., vol. 9, no. 2, pp. 1381-1384, June 1999.

[8] — "Over-voltage suppression in a fault current limiter by a $\mathrm{ZnO}$ varistor," IEEE Trans. Appl. Supercond., vol. 13, no. 1, pp. 880-883, June 2003. 